\title{
Uso de Dexmedetomidina em Anestesia Geral para Tratamento Cirúrgico de Aneurisma Cerebral, em Paciente Gestante, Portadora de Doença Hipertensiva Específica da Gestação. Relato de Caso *
}

\section{Dexmedetomidine in General Anesthesia for Surgical Treatment of Cerebral Aneurysm in Pregnant Patient with Specific Hypertensive Disease of Pregnancy. Case Report}

\author{
Kleber Machareth de Souza ${ }^{1}$; Luiz César Anzoategui, TSA ${ }^{2}$, \\ Washington Cássio Justino Pedroso, TSA ${ }^{1}$; Werner Alfred Gemperli, TSA ${ }^{1}$
}

\begin{abstract}
RESUMO
Souza KM, Anzoategui LC, Pedroso WCJ, Gemperli WA - Uso de Dexmedetomidina em Anestesia Geral para Tratamento Cirúrgico de Aneurisma Cerebral, em Paciente Gestante, Portadora de Doença Hipertensiva Específica da Gestação. Relato de Caso
\end{abstract}

JUSTIFICATIVA E OBJETIVOS: A incidência de cirurgias não obstétricas em pacientes gestantes é de $0,36 \%$ a $2 \%$. No entanto, cirurgias visando o tratamento cirúrgico de aneurisma cerebral em gestantes são extremamente raras. A doença hipertensiva específica da gestação, apresenta prevalência clínica de $10 \%$ na população gestante. Trata-se de uma doença de elevada complexidade clínica, acometendo múltiplos órgãos e sistemas. $A$ dexmedetomidina, fármaco agonista $\alpha_{2}$, apresenta importante seletividade para estes receptores, quando utilizada em doses clínicas terapêuticas e promove adequada estabilidade hemodinâmica, se empregada no período peri-operatório. O objetivo deste relato foi apresentar uma técnica com a qual fosse possível a manutenção da homeostase materna, preservando ao máximo o fluxo sangüíneo útero-placentário e a vitalidade fetal, sem deixar de lado aspectos fundamentais relativos à otimização da relação oferta/demanda de oxigênio cerebral e adequação das condições do tecido cerebral propícias ao manuseio cirúrgico.

RELATO DO CASO: Gestante com 19 anos encaminhada para tratamento cirúrgico de aneurisma cerebral, estando na vigésima sétima semana de gestação. No pré-operatório, apresentava-se consciente, orientada, com presença de déficit à esquerda e quadro clínico compatível com toxemia gravídica. Foi administrada dexmedetomidina $\left(1 \mu \mathrm{g} \cdot \mathrm{kg}^{-1}\right)$ em 20 minutos, seguida de indução anestésica com propofol $\left(2,5 \mathrm{mg} \cdot \mathrm{kg}^{-1}\right)$, fentanil $\left(7,5 \mu \mathrm{g} \cdot \mathrm{kg}^{-1}\right)$, lidocaína (1 $\left.\mathrm{mg}^{\mathrm{kg}} \mathrm{kg}^{-1}\right)$ e rocurônio $\left(2 \mathrm{mg} \cdot \mathrm{kg}^{-1}\right)$ em seqüência rápida. A manutenção da anestesia foi obtida com propofol $\left(50 \mu \mathrm{g} \cdot \mathrm{kg}^{-1} \cdot \mathrm{min}^{-1}\right)$, alfentanil $\left(1 \mu \mathrm{g} \cdot \mathrm{kg}^{-1} \cdot \mathrm{min}^{-1}\right)$ e dexmedetomidina $\left(0,7 \mu \mathrm{g} \cdot \mathrm{kg}^{-1} \cdot \mathrm{min}^{-1}\right)$. A cirurgia foi realizada sem qualquer intercorrência, não havendo seqüela neurológica subjacente.

CONCLUSÕES: Neste caso o uso da dexmedetomidina tornou possível um adequado manuseio hemodinâmico, mantendo otimizado o fluxo sangüíneo útero-placentário e a vitalidade fetal.

\footnotetext{
${ }^{*}$ Recebido do (Received from) CET da Santa da Casa de Campo Grande, MS

1. Anestesiologista do Serviço de Anestesiologia da Santa Casa de Campo Grande

2. Anestesiologista Responsável pelo CET da Santa Casa de Campo Grande

Apresentado (Submitted) em 26 de abril de 2004
}

Aceito (Accepted) para publicação em 08 de dezembro de 2004

Endereço para correspondência (Correspondence to)

Dr. Kleber Machareth de Souza

Rua Pernambuco, 946/403 Bairro Monte Castelo

79010-040 Campo Grande, MS

E-mail:machareth@terra.com.br

(C) Sociedade Brasileira de Anestesiologia, 2005
Ressaltam-se, ainda, as condições adequadas de manuseio cirúrgico do tecido cerebral, assim como a ausência de influência na morbidade após o procedimento anestésico-cirúrgico.

Unitermos: ANESTESIA, Geral; CIRURGIA, Neurocirurgia: aneurisma; DOENÇAS: hipertensiva específica da gravidez; DROGAS: dexmedetomidina

\section{SUMMARY}

Souza KM, Anzoategui LC, Pedroso WCJ, Gemperli WA - Dexmedetomidine in General Anesthesia for Surgical Treatment of Cerebral Aneurysm in Pregnant Patient with Specific Hypertensive Disease of Pregnancy. Case Report

BACKGROUND AND OBJECTIVES: The incidence of non-obstetrical surgeries in pregnant patients is about $0.36 \%$ to $2 \%$. However, surgeries aiming at surgical treatment of cerebral aneurysm in pregnant women are extremely rare. Specific hypertensive disease of pregnancy, shows clinical prevalence of $10 \%$. It is a disease with high clinical complexity compromising multiple organs and systems. Dexmedetomidine, $\alpha_{2}$-adrenoceptor agonist drug, in therapeutic clinical doses has major selectivity for these receptors and promotes suitable hemodynamic stability if used in the preoperative period. The purpose of this report was to present an anesthetic technique able to provide adequate maintenance of maternal homeostasis, preserving to the highest level uterus-placental blood flow and fetal vitality, without neglecting fundamental aspects regarding the optimization of brain oxygen supply/demand ratio and favorable brain tissue conditions for surgical management.

CASE REPORT: Pregnant patient, 19 years old, 27 weeks of gestation, was referred to the operating room for surgical treatment of cerebral aneurysm. In the preoperative period she was conscious, oriented, eupneic but with left side motor deficit and clinical signs compatible with toxemia of pregnancy. Dexmedetomidine $\left(1 \mu \mathrm{g} \cdot \mathrm{kg}^{-1} \cdot \mathrm{h}^{-1}\right)$ was administered in 20 minutes, followed by anesthetic induction with propofol $\left(2.5 \mathrm{mg}^{-\mathrm{kg}^{-1}}\right)$, fentanyl $\left(7.5 \mu \mathrm{g} \cdot \mathrm{kg}^{-1}\right)$, lidocaine $\left(1 \mathrm{mg}^{\mathrm{kg}} \mathrm{g}^{-1}\right)$ and rocuronium $\left(1.2 \mathrm{mg}^{\mathrm{kg}} \mathrm{kg}^{-1}\right.$ ) in rapid sequence. Anesthesia was maintained with propofol $\left(50 \mu \mathrm{g} \cdot \mathrm{kg}^{-1} \cdot \mathrm{min}^{-1}\right)$, alfentanil $\left(1 \mu \mathrm{g} \cdot \mathrm{kg}^{-1} \cdot \mathrm{min}^{-1}\right)$ and dexmedetomidine $\left(0.7 \mu \mathrm{g} \cdot \mathrm{kg}^{-1} \cdot \mathrm{h}^{-1}\right)$. Surgical procedure went on with no complications, including brain sequelae.

CONCLUSIONS: This case report has shown that dexmedetomidine made possible the handling of hemodynamic responses, keeping optimized uterus-placental blood flow and fetal vitality. Adequate conditions of surgical brain tissue manipulation and the absence of influence in postoperative morbidity are also emphasized.

Key Words: ANESTHESIA, General; DISEASES: specific hypertensive pregnancy; DRUGS: dexmedetomidine; SURGERY, Neurosurgical: aneurysm 


\section{INTRODUÇÃO}

$\mathrm{O}$ tratamento cirúrgico de doenças neurológicas durante o período gestacional é extremamente infreqüente na prática clínica. O período gestacional e as alterações fisiológicas que a ele se sobrepõe por si só já preconizam cuidados especiais. A presença de doença hipertensiva específica da gestação representa um desafio adicional no tocante ao manuseio anestésico dessas pacientes.

Na literatura internacional, a doença hipertensiva específica da gestação manifesta-se em $7 \%$ a $10 \%$ de toda a população gestante, podendo ser classificada como leve ou grave. Dentre as principais causas de morbimortalidade encontram-se os acidentes vasculares encefálicos ${ }^{1}$.

Clinicamente ela se caracteriza como um estado generalizado de vasoconstrição e hipoperfusão, com retenção de sódio, água e edema. Entretanto, o espaço intravascular encontra-se diminuído. O grau de comprometimento hemodinâmico da paciente caracteriza a gravidade da doença; hipertensão arterial, aumento da freqüência cardíaca e da resistência vascular periférica estão presentes em graus variados. Pode ocorrer comprometimento do fluxo sangüíneo útero-placentário, hepático, renal e do sistema nervoso central, sendo a proteinúria um achado característico da doença. Trata-se, portanto, de alteração sistêmica de complexo controle clínico ${ }^{1}$.

A manutenção da estabilidade hemodinâmica, o controle rigoroso da pressão intracraniana, a proteção encefálica com adequação da relação oferta/demanda de oxigênio, a manutenção de boa complacência do tecido encefálico frente ao manuseio cirúrgico e o despertar precoce após o término do procedimento - desde que possível - permitindo avaliação neurológica precoce da paciente, constituem-se os principais objetivos da técnica anestésica em neurocirurgia ${ }^{2}$.

Adexmedetomidina, fármaco agonista alfa ${ }_{2}$-adrenérgico dotado de ação sedativa e analgésica, introduzido na prática clínica anestesiológica como agente adjuvante de natureza analgésica e hipnótico-sedativa, é capaz de promover eficiente estabilidade hemodinâmica, reduzir o consumo de anestésicos e promover despertar precoce e suave, com menor risco de depressão respiratória, evitando, assim, retenção de $\mathrm{CO}_{2}$ e, conseqüentemente, aumento da $\mathrm{PaCO}_{2}$, fato este indesejável no pós-operatório neurocirúrgico ${ }^{3,4}$.

O objetivo deste relato é apresentar o caso clínico de uma gestante (27 semanas), portadora de doença hipertensiva específica da gestação, a ser submetida a tratamento cirúrgico de aneurisma encefálico.

\section{RELATO DO CASO}

Paciente com 19 anos, 58 kg, $168 \mathrm{~cm}$, na vigésima sétima semana de gestação, com quadro clínico compatível de doença hipertensiva específica da gestação. Foi encaminhada à sala operatória apresentando déficit motor à esquerda decorrente de acidente vascular encefálico hemorrágico em artéria comunicante posterior, mantendo-se consciente, orien- tada auto e halopsiquicamente, sem a presença de qualquer outro sinal clínico neurológico.

Apresentava ainda pressão arterial de $170 \times 110 \mathrm{mmHg}$, freqüência cardíaca de 128 batimentos por minuto e edema nos membros inferiores ++/++++. Exames complementares mostravam proteinúria de $4,5 \mathrm{~g} .24 \mathrm{~h}^{-1}$, com bioquímica sangüínea e provas de função hepática normais. A paciente foi monitorizada com ECG na derivação $D_{\text {II }}$, oximetria de pulso, capnografia, pressão arterial média invasiva na artéria radial esquerda e pressão venosa central. A medicação pré-anestésica foi feita com dexmedetomidina $\left(1 \mu \mathrm{g} . \mathrm{kg}^{-1}\right)$ em 20 minutos. A anestesia foi induzida com propofol $\left(2,5 \mathrm{mg} . \mathrm{kg}^{-1}\right)$, fentanil $\left(7,5 \mu \mathrm{g} \cdot \mathrm{kg}^{-1}\right)$, lidocaína $\left(1 \mathrm{mg} \cdot \mathrm{kg}^{-1}\right)$ e rocurônio $(1,2$ $\left.\mathrm{mg} \cdot \mathrm{kg}^{-1}\right)$ em seqüência rápida e sob manobra de Sellick, atéa intubação traqueal. A manutenção da anestesia se deu com propofol $\left(50 \mu \mathrm{g} \cdot \mathrm{kg}^{-1} \cdot \mathrm{min}^{-1}\right)$, alfentanil $\left(1 \mu \mathrm{g} \cdot \mathrm{kg}^{-1} \cdot \mathrm{min}^{-1}\right)$, rocurônio $\left(6 \mu \mathrm{g} \cdot \mathrm{kg}^{-1} \cdot \mathrm{min}^{-1}\right)$ e dexmedetomidina $\left(0,7 \mu \mathrm{g} \cdot \mathrm{kg}^{-1} \cdot \mathrm{h}^{-1}\right)$, sendo que a infusão desta última foi reduzida gradualmente até $0,4 \mu \mathrm{g} \cdot \mathrm{kg}^{-1} \cdot \mathrm{h}^{-1}$, de acordo com os parâmetros clínicos avaliados (pressão arterial média por método invasivo e freqüência cardíaca). A hidratação intra-operatória foi feita com solução fisiológica a $0,9 \%$ na velocidade de $6 \mathrm{ml} \cdot \mathrm{kg}^{-1} \cdot \mathrm{h}^{-1}$, após reposição do jejum pré-operatório. Realizou-se gasometria arterial e bioquímica sangüínea completa a cada 60 minutos. A paciente foi mantida em ligeiro decúbito lateral esquerdo durante o procedimento cirúrgico, sendo realizada monitorização periódica do batimento cardíaco fetal com sonar. Manteve-se hemodinamicamente estável (pressão arterial em torno de $110 \times 60 \mathrm{mmHg}$ e pressão arterial média em torno de $76 \mathrm{mmHg}$ ) durante os 320 minutos do procedimento anestésico-cirúrgico, apresentando boas condições para manipulação cirúrgica do tecido encefálico. Ao término do procedimento, a paciente foi encaminhada à UTI, sendo extubada 90 minutos após o final da cirurgia, sem qualquer intercorrência ou seqüela neurológica subjacente. A vitalidade fetal manteve-se inalterada durante todo o período. Após 10 semanas da intervenção cirúrgica, a paciente foi submetida à operação cesariana sem qualquer intercorrência, tanto para mãe, como para o concepto.

\section{DISCUSSÃO}

Este relato de caso teve como objetivo apresentar uma técnica anestésica capaz de preservar a homeostase materna, favorecer aspectos fundamentais inerentes à técnica neurocirúrgica com manutenção da relação oferta/demanda de oxigênio encefálico ${ }^{1} \mathrm{e}$, na medida do possível, preservar ao máximo o fluxo sangüíneo útero-placentário.

A dexmedetomidina, agente agonista dos receptores alfa $_{2}$-adrenérgicos e empregada como adjuvante aos agentes anestésicos, conferiu grande estabilidade hemodinâmica à paciente ${ }^{3}$. Estudos relativamente recentes têm demonstrado que a dexmedetomidina é capaz de reduzir em torno de $50 \%$ a concentração plasmática de norepinefrina e epinefrina circulante ${ }^{4}$. Alguns trabalhos têm evidenciado que a dexmedetomidina reduz o fluxo sangüíneo encefálico por ação direta nos receptores alfa 2 pós-sinápticos ${ }^{5,6}$, além 
de sua capacidade intrínseca em reduzir a pressão liquórica ${ }^{7}$. Quando se infere indiretamente seus efeitos hemodinâmicos e sua ação sobre a vascularização encefálica, pode-se admitir que este fármaco é capaz de reduzir efetivamente o metabolismo encefálico e a pressão intracraniana ${ }^{8,9}$. Não está bem estabelecido na literatura se a dexmedetomidina é capaz de reduzir efetivamente em humanos o metabolismo encefálico. Estudos recentes em animais mostram que a dexmedetomidina é capaz de promover redução do fluxo sangüíneo encefálico de até $45 \%$, sem que haja sofrimento isquêmico ${ }^{10}$. Outros estudos têm demonstrado ser a dexmedetomidina capaz de promover redução da lesão causada por isquemia focal ${ }^{11}$.

Ainda são necessárias pesquisas mais consistentes em relação ao possível efeito neuroprotetor da dexmedetomidina em humanos, bem como sua ação farmacológica conjunta frente ao binômio mãe-feto ${ }^{12}$. Porém, sem dúvida alguma ficou evidente seu benefício frente a este relato de caso, promovendo adequada estabilidade hemodinâmica (PAM = 76 $\mathrm{mmHg} \pm 10 \mathrm{mmHg}$ e $\mathrm{FC}=75 \mathrm{bpm} \pm 10 \mathrm{bpm}$ ) e sensível redução global no consumo de fármacos anestésicos.

Houve redução de $70 \%$ dos valores iniciais infundidos de alfentanil e $50 \%$ dos valores iniciais infundidos de propofol, associados à redução de $43 \%$ na taxa de infusão de dexmedetomidina $\left(0,7 \mu \mathrm{g} \cdot \mathrm{kg}^{-1} \cdot \mathrm{min}^{-1}\right.$ para $\left.0,4 \mu \mathrm{g} \cdot \mathrm{kg}^{-1} \cdot \mathrm{min}^{-1}\right)$.

$\mathrm{O}$ presente caso mostrou que o uso da dexmedetomidina permitiu um adequado manuseio hemodinâmico da paciente, condições otimizadas de manuseio cirúrgico do tecido encefálico, assim como ausência de influência na morbidade do binômio mãe-feto após o procedimento anestésico-cirúrgico.

\section{Dexmedetomidine in General Anesthesia for Surgical Treatment of Cerebral Aneurysm in Pregnant Patient with Specific Hypertensive Disease of Pregnancy. Case Report}

Kleber Machareth de Souza, M.D.; Luiz César Anzoategui, TSA, M.D.; Washington Cássio Justino Pedroso, TSA, M.D.; Werner Alfred Gemperli, TSA, M.D.

\section{INTRODUCTION}

Surgical treatment of neurological diseases during pregnancy is extremely uncommon. Gestation and related physiological changes per se require special care. The association of specific hypertensive disease of pregnancy is an additional challenge in terms of anesthetic management of these patients.

In the international literature, specific hypertensive disease of pregnancy is manifested in $7 \%$ to $10 \%$ of all pregnant popu- lation and may be classified as mild or severe. Strokes are among major causes of morbidity/mortality ${ }^{1}$.

Clinically, it is characterized by generalized vasoconstriction and hypoperfusion, with sodium and water retention and edema. Intravascular space, however, is decreased. The level of hemodynamic impairment characterizes the severity of the disease; hypertension, increased heart rate and peripheral vascular resistance are present in different levels. There may be uterus-placental, liver, renal and central nervous system blood flow impairment being proteinuria a typical finding of the disease. So, it is a systemic change with complex clinical control ${ }^{1}$. Hemodynamic stability, rigorous control of intracranial pressure, brain protection with adequate oxygen supply/demand ratio, maintenance of adequate brain tissue compliance during surgical procedure and early postoperative emergence - if possible - allowing for early neurological evaluation, are major objectives of neurosurgical anesthetic techniques ${ }^{2}$.

Dexmedetomidine, an $\alpha_{2}$ adrenoceptor agonista drug with sedative and analgesic properties and introduced in the clinical practice as adjuvant analgesic, hypnotic and sedative agent, is able to promote efficient hemodynamic stability, decrease anesthetic consumption and promote early and smooth emergence with lower respiratory depression risk, thus preventing $\mathrm{CO}_{2}$ retention and, as a consequence, increased $\mathrm{PaCO}_{2}$, which is undesirable during neurosurgical postoperative period $^{3,4}$.

This report aimed at presenting the clinical case of a pregnant patient (27 weeks), with specific hypertensive disease of pregnancy, to be submitted to surgical treatment of cerebral aneurysm.

\section{CASE REPORT}

Female patient, 19 years old, $58 \mathrm{~kg}, 168 \mathrm{~cm}, 27$ weeks of gestation, with clinical symptoms compatible with specific hypertensive disease of pregnancy. Patient was referred to the operating room with left side motor deficit caused by hemorrhagic stroke in posterior communicating artery, however conscious, oriented and without any other neurological sign. Patient also presented blood pressure $=170 \times 110 \mathrm{mmHg}$, heart rate $=128$ beats per minute and lower limbs edema $++/++++$. Additional tests have shown $4.5 \mathrm{~g} .24 \mathrm{~h}^{-1}$ proteinuria with normal blood biochemistry and liver function. Patient was monitored with ECG at $D_{\|}$lead, pulse oximetry, capnography, invasive blood pressure in left radial artery and central venous pressure. Patient was premedicated with dexmedetomidine $\left(1 \mu \mathrm{g} . \mathrm{kg}^{-1}\right)$ in 20 minutes. Anesthesia was induced with propofol $\left(2.5 \mathrm{mg} \cdot \mathrm{kg}^{-1}\right)$, fentanyl $\left(7.5 \mu \mathrm{g} \cdot \mathrm{kg}^{-1}\right)$, lidocaine $\left(1 \mathrm{mg} \cdot \mathrm{kg}^{-1}\right)$ and rocuronium $\left(1.2 \mathrm{mg} \cdot \mathrm{kg}^{-1}\right)$ in rapid sequence under Sellick's maneuver until tracheal intubation. Anesthesia was maintained with propofol $\left(50 \mu \mathrm{g} \cdot \mathrm{kg}^{-1} \cdot \mathrm{min}^{-1}\right)$, alfentanil $\left(1 \mu \mathrm{g} \cdot \mathrm{kg}^{-1} \cdot \mathrm{min}^{-1}\right)$, rocuronium $\left(6 \mu \mathrm{g} \cdot \mathrm{kg}^{-1} \cdot \mathrm{min}^{-1}\right)$ and dexmedetomidine $\left(0.7 \mu \mathrm{g} \cdot \mathrm{kg}^{-1} \cdot \mathrm{h}^{-1}\right)$. Dexmedetomidine infusion was gradually decreased to $0.4 \mu \mathrm{g} \cdot \mathrm{kg}^{-1} \cdot \mathrm{h}^{-1}$, according to evaluated parameters (invasive mean blood pressure and heart rate). 
Intraoperative hydration was achieved with saline at the rate of $6 \mathrm{~mL} \cdot \mathrm{kg}^{-1} \cdot \mathrm{h}^{-1}$, after preoperative fast replacement. Arterial gases and blood biochemistry were analyzed every 60 minutes. Patient was maintained in slight left lateral position during surgery and fetal heart rate was periodically monitored by sonar. Patient was maintained hemodynamically stable (blood pressure around $110 \times 60 \mathrm{mmHg}$ and mean blood pressure around $76 \mathrm{mmHg}$ ) during the 320 minutes of procedure, with good conditions for surgical brain tissue manipulation.

Patient was referred to the ICU at procedure completion and was extubated 90 minutes after surgery without intercurrences or underlying neurological sequelae. Fetal vitality was maintained unchanged throughout the period. Patient was submitted to Cesarean section 10 weeks later without any intercurrence both for mother and fetus.

\section{DISCUSSION}

This report aimed at presenting an anesthetic technique able to preserve maternal homeostasis, to favor fundamental aspects inherent to neurosurgical technique with maintenance of brain oxygen supply/demand ratio and, whenever possible ${ }^{1}$, to preserve at the utmost uterus-placental blood flow. Dexmedetomidine, $\alpha_{2}$ adrenoceptor agonist used as adjuvant to anesthetic agents, has provided major hemodynamic stability ${ }^{3}$. Relatively recent studies have shown that dexmedetomidine is able to decrease circulating plasma norepinephrine and epinephrine concentration in approximately $50 \%{ }^{4}$. Some studies have shown that dexmedetomidine decreases brain blood flow by directly acting on post-synaptic $\alpha_{2}$ receptors ${ }^{5,6}$, in addition to its intrinsic ability to decrease CSF pressure ${ }^{7}$. When its hemodynamic effects and action on brain vascularization are indirectly assumed, one may admit that this drug is able to effectively decrease brain metabolism and intracranial pressure ${ }^{8,9}$. Dexmedetomidine ability to decrease brain metabolism in humans is still not well established by the literature. Recent animal studies have shown that dexmedetomidine is able to decrease blood flow in up to $45 \%$ without ischemic suffering ${ }^{10}$. Other studies have shown that dexmedetomidine is able to decrease injury caused by focal ischemia ${ }^{11}$.

More consistent studies on possible dexmedetomidine neuroprotective effect in humans, as well as its joint pharmacological action on the binomial mother-fetus are still needed

${ }^{12}$. However, its benefits in this case report by promoting adequate hemodynamic stability (MBP $=76 \mathrm{mmHg} \pm 10 \mathrm{mmHg}$ and $\mathrm{HR}=75 \mathrm{bpm} \pm 10 \mathrm{bpm}$ ) and decreasing anesthetic drugs consumption are undoubtedly apparent.

There has been $70 \%$ decrease in alfentanil initial values and $50 \%$ in propofol initial values, associated to $43 \%$ decrease in dexmedetomidine infusion rate $\left(0.7 \mu \mathrm{g} \cdot \mathrm{kg}^{-1} \cdot \mathrm{min}^{-1}\right.$ to 0.4 $\mu \mathrm{g} \cdot \mathrm{kg}^{-1} \cdot \mathrm{min}^{-1}$ )

This case has shown that dexmedetomidine has allowed for adequate hemodynamic handling, optimized surgical handling of brain tissue, as well as the absence of influence in morbidity of the binomial mother-fetus after the anesthetic-surgical procedure.

\section{REFERÊNCIAS - REFERENCES}

01. Miller RD, Cucchiara RF, Miller ED et al - Anesthesia. $5^{\text {th }}$ Ed, Philadelphia, Churchill Livingstone, 2000;2:2053-2055.

02. Albin MS, Albers JW, Albin RL et al - Textbook of Neuroanesthesia: with Neurosurgical and Neuroscience Perspective. $1^{\text {st }}$ Ed, New York, The McGraw-Hill Companies, 1997;21-44.

03. Ebert TJ, Hall JE, Barney JA et al - The effects of increasing plasma concentration of dexmedetomidine in humans. Anesthesiology, 2000;93:382-394.

04. Talke P, Li J, Jain U - Effects of per operative dexmedetomidine infusion in patients undergoing vascular surgery. Anesthesiology, 1995;82:620-633.

05. Karlsson BR, Forsman M, Roald OK - Effect of dexmedetomidine, a selective and potent alfa 2-agonist, on cerebral blood flow and oxygen consumption during halothane anesthesia in dogs. Anesth Analg, 1990;71:125-129.

06. Coughlan MG, Lee JG, Bosnjak ZJ et al - Direct coronary and cerebral vascular responses to dexmedetomidine. Significance of endogenous nitric oxide synthesis. Anesthesiology, 1992;77:998-1006.

07. Talke P, Tong C, Lee HW et al - Effect of dexmedetomidine on lumbar cerebrospinal fluid pressure in humans. Anesth Analg, 1997;85:358-364.

08. Zornow MH, Scheller MS, Sheehan PB - Intracranial pressure effects of dexmedetomidine in rabbits. Anesth Analg,1992;75:232-237.

09. Favre JB, Gardaz JP, Ravussin P - Effect of clonidine on ICP on the hemodynamic responses to nociceptive stimuli in patients with brain tumors. J Neurosurg Anesthesiol, 1995;7:159-167.

10. Zornow MH, Fleischer JE, Scheller MS - Dexmedetomidine, an alpha 2-adrenergic-agonist, decreases cerebral blood flow in the isoflurane-anesthetized dogs. Anesth Analg, 1990;70: 624-630.

11. Maier C, Steinberg GK, Sun GH - Neuroprotection by the alpha 2-adrenoreceptor agonist dexmedetomidine in a focal model of cerebral ischemia. Anesthesiology, 1993;79:306-312.

12. Aho M, Erkola O, Kallio A et al - Dexmedetomidine infusion for maintenance of anesthesia in patients undergoing abdominal hysterectomy. Anesth Analg, 1992;75:940-946.

\section{RESUMEN}

Souza KM, Anzoategui LC, Pedroso WCJ, Gerperli WA- Uso de Dexmedetomidina en Anestesia General para Tratamiento Quirúrgico de Aneurisma Cerebral, en Paciente Embarazada, Portadora de Enfermedad Hipertensiva Específica del Embarazo. Relato de Caso

JUSTIFICATIVA Y OBJETIVOS: La incidencia de cirugías no obstétricas en pacientes embarazadas es del 0,36\% a 2\%. Sin embargo, cirugías con la finalidad de tratamiento quirúrgico de aneurisma cerebral en embarazadas son extremadamente raras. La enfermedad hipertensiva específica de la gestación, presenta superioridad clínica del $10 \%$ en la población gestante. Se trata de una enfermedad de elevada complejidad clínica, acometiendo múltiples órganos y sistemas. La dexmedetomidina, fármaco agonista $\alpha-2$, presenta importante selectividad para estos receptores cuando utilizada en dosis clínicas terapéuticas y promueve adecuada estabilidad hemodinámica cuando empleada en el período peri-operatorio. El objetivo de este relato fue presentar una técnica con la cual hubiera sido posible el mantenimiento de la homeostasis materna, 
preservando al máximo el flujo sanguíneo útero-placentario y la vitalidad fetal, sin dejar de lado aspectos fundamentales relativos a la optimización de la relación oferta/demanda de oxígeno cerebral y adecuación de las condiciones del tejido cerebral propicias al manoseo quirúrgico.

RELATO DEL CASO: Embarazada con 19 años encaminada para tratamiento quirúrgico de aneurisma cerebral, en la vigésima séptima semana de embarazo. En el pre-operatorio, se presentaba consciente, orientada, con presencia de déficit a la izquierda y cuadro clínico compatible con toxemia gravídica. Fue administrada dexmedetomidina $\left(1 \mu \mathrm{g} . \mathrm{kg}^{-1}\right)$ en 20 minutos, seguida de inducción anestésica con propofol $\left(2,5 \mathrm{mg}^{\mathrm{kg}} \mathrm{k}^{-1}\right)$, fentanil $\left(7,5 \mu \mathrm{g} \cdot \mathrm{kg}^{-1}\right)$, lidocaína $\left(1 \mathrm{mg} \cdot \mathrm{kg}^{-1}\right)$ y rocurônio
(2 $\left.\mathrm{mg} \cdot \mathrm{kg}^{-1}\right)$ en secuencia rápida. El mantenimiento de la anestesia fue lograda con propofol $\left(50 \mu \mathrm{g} \cdot \mathrm{kg}^{-1} \cdot \mathrm{min}^{-1}\right)$, alfentanil (1 $\mu \mathrm{g} \cdot \mathrm{kg}^{-1} \cdot \mathrm{min}^{-1}$ ) y dexmedetomidina $\left(0,7 \mu \mathrm{g} \cdot \mathrm{kg}^{-1} \cdot \mathrm{min}^{-1}\right)$. La cirugía fue realizada sin cualquier acontecimiento, no habiendo secuela neurológica subyacente.

CONCLUSIONES: El presente caso mostró que el uso de la dexmedetomidina posibilitó un adecuado manoseo hemodinámico, manteniendo optimizado el flujo sanguíneo útero-placentario y la vitalidad fetal. Se resaltan aún las condiciones adecuadas de manipulación quirúrgica del tejido cerebral, así como la ausencia de influencia en la morbidad después del procedimiento anestésico-quirúrgico. 